\title{
A Mathematical and Numerically Integrable Modeling of 3D Object Grasping under Rolling Contacts between Smooth Surfaces
}

\author{
Suguru Arimoto and Morio Yoshida \\ RIKEN-TRI Collaboration Center for Human-Interactive Robot Research, Nagoya, Aichi 463-0003, Japan \\ Correspondence should be addressed to Morio Yoshida, yoshida@nagoya.riken.jp
}

Received 31 March 2011; Accepted 25 July 2011

Academic Editor: Antonio Munjiza

Copyright (c) 2011 S. Arimoto and M. Yoshida. This is an open access article distributed under the Creative Commons Attribution License, which permits unrestricted use, distribution, and reproduction in any medium, provided the original work is properly cited.

A computable model of grasping and manipulation of a 3D rigid object with arbitrary smooth surfaces by multiple robot fingers with smooth fingertip surfaces is derived under rolling contact constraints between surfaces. Geometrical conditions of pure rolling contacts are described through the moving-frame coordinates at each rolling contact point under the postulates: (1) two surfaces share a common single contact point without any mutual penetration and a common tangent plane at the contact point and (2) each path length of running of the contact point on the robot fingertip surface and the object surface is equal. It is shown that a set of Euler-Lagrange equations of motion of the fingers-object system can be derived by introducing Lagrange multipliers corresponding to geometric conditions of contacts. A set of 1st-order differential equations governing rotational motions of each fingertip and the object and updating arc-length parameters should be accompanied with the Euler-Lagrange equations. Further more, nonholonomic constraints arising from twisting between the two normal axes to each tangent plane are rewritten into a set of Frenet-Serre equations with a geometrically given normal curvature and a motion-induced geodesic curvature.

\section{Introduction}

In relation to the recent development of robotics research and neurophysiology, there arises an important question on a study of the functionality of the human hand in grasping and object manipulation interacting physically with environment under arbitrary geometries of objects and fingertips. Another question also arises as to whether a complete mathematical model of grasping a $3 \mathrm{D}$ rigid object with an arbitrary shape can be developed and used in numerical simulation to validate control models of prehensile functions of a set of multiple fingers. In particular, is it possible to develop a mathematical model as a set of Euler-Lagrange equations that govern a whole motion of the fingers-object system under rolling contact constraints between each robot fingertip and a rigid object with an arbitrary smooth surface.

Traditionally in robotics research, a rolling contact constraint between two rigid-body surfaces is defined as the zero velocity of one translational motion of the common contact point on the fingertip surface relative to another on the object surface [1]. Therefore, rolling contact constraints are expressed in terms of velocity relations called a Pfaffian form. Montana [2] presented a complete set of all velocity relations of a rolling contact by using the normalized gauss frame for expressing given smooth surfaces of fingertips and a 3D object. Based on Montana's set of Pfaffian forms, Paljug et al. [3] formulated a dynamic model for the control of rolling contacts in multiarm manipulation. However, it is uncertain whether the derived model of equations of motion can be computationally integrable in time in case of rolling contacts between general smooth surfaces, since in [3] only a limited case of ball-plate contacts was numerically simulated. Another work by Cole et al. [4] tried to simulate a 3D grasping, but it is uncertain whether it could overcome the problem of arise of a nonholonomic constraint pointed out by Montana [2].

Even in case of 2D grasping by means of dual robot fingers with smooth fingertip surfaces, the integrability of 
Pfaffian forms of rolling contact constraints was shown very recently in our previous paper [5], where a complete set of computational models of Euler-Lagrange equations of motion of the whole fingers-object system and a pair of firstorder differential equations expressing update laws of arclength parameters along smooth contour curves of the object were given. Instead of the zero relative-velocity assumption of rolling contact, the following set of postulates for pure rolling contact is introduced:

(1) two contact points on each contour curve must coincide at a single common point without mutual penetration,

(2) the two contours must have the same tangent at the common contact.

Owing to these postulates, the path length of one contact point running on each fingertip contour curve and that of another contact point running on the object contour must coincide, that is, the constraint can be expressed eventually in the level of position variable. Hence, it is shown in [6] that Pfaffian constraints are integrable, and their integral forms are derived explicitly by using the moving frame coordinates. It is further shown [6] that the quantities of the second fundamental form of concerned contour curves do not appear in the Euler-Lagrange equations but play a key role in the update laws of arc-length parameters of the curves.

This paper aims at extending such a moving frame coordinates approach for mathematical modelling of $2 \mathrm{D}$ grasping to computable mathematical modelling of $3 \mathrm{D}$ grasping of a rigid object with arbitrary smooth surfaces under the following set of $3 \mathrm{D}$ rolling contact constraints:

(a1) two contact points on each curved surface must coincide at a single common point without mutual penetration,

(a2) the two curved surfaces have the same tangent at the common contact point, that is, each surface has the same unit normal with mutually opposite direction at the common contact point,

(a3) the two path lengths running on their corresponding surfaces must be coincident.

In the previous paper [7], a set of Euler-Lagrange equations of motion of the fingers-object system have been derived by using the moving frame coordinates, but any explicit set of update laws of moving frame coordinates have been not given yet. In particular, any mathematical role of the quantities of the second fundamental form of a contact curve running on a concerned surface has not yet been studied in a mathematically explicit way. Therefore, it still remains unsolved to construct a complete set of equations of $3 \mathrm{D}$ grasping under rolling contact constraints in the situation of arbitrary given geometry of surfaces. This paper shows that nonholonomic constraints arising from relative twisting among the two normal axes at the contact point can be naturally resolved into determination of each of geodesic curvatures of the curves of the contact point on the fingertip surface and the object surface. Another second fundamental form of normal curvature on each surface is assumed to be extracted from a data structure of a given rigid body object, together with that of unit normal at each specified point on its surface. Thus, a set of 3D Frenet-Serret equations with normal and geodesic curvatures that update the moving frame coordinates are determined and shown to be computationally integrable together with the set of EulerLagrange equations of motion of the whole system.

\section{Preliminary Results on Derivation of Euler-Lagrange Equations}

Consider firstly a physical situation that a pair of multijoint robot fingers is grasping a $3 \mathrm{D}$ rigid body as seen in Figure 1. In this figure, the inertial frame denoted by $O-x y z$ is fixed in the Euclidean space $\mathbf{E}^{3}$, and local coordinates systems denoted by $O_{i}-X_{i} Y_{i} Z_{i}$ for $i=1,2$ are introduced at each robot fingerend. The local coordinates system of the object is denoted by $O_{m}-X Y Z$ as shown in Figure 1, where $O_{m}$ stands for the object mass center. Next, denote each locus of points of contact between the two surfaces by a curve $\gamma_{i}\left(s_{i}\right)$ (3dimensional vector in $\mathrm{E}^{3}$ ) with length parameter $s_{i}$ on its corresponding surface $S_{i}(i=0,1)$, where $i=0$ signifies the object, and $i=1$ does the left hand fingerend. It is possible to assume that, given a curve $\gamma_{1}\left(s_{1}\right)$ as a locus of points of contact on $S_{1}$ and another curve $\gamma_{0}\left(s_{0}\right)$ as a locus of contact points on $S_{0}$, the two curves coincide at contact point $P_{1}$ and share the same tangent plane $T_{1}$ at $P_{1}$ (see Figure 2). Further, during continuation of rolling contact, the two curves $\gamma_{0}\left(s_{0}\right)$ and $\gamma_{1}\left(s_{1}\right)$ can be described in terms of the same length parameter $s$ in such a way that $s_{0}=s+c_{0}$ and $s_{1}=s+c_{1}$, where $c_{0}$ and $c_{1}$ are constant.

Second, suppose that at some $s$ of the length parameter the two curves $\gamma_{0}\left(s_{0}\right)$ and $\gamma_{1}\left(s_{1}\right)$ coincide at $P_{1}(s)$. Since $\gamma_{0}\left(s_{0}\right)$ is described in local coordinates $O_{m}-X Y Z$, its expression in the frame coordinates is given by

$$
\bar{\gamma}_{0}\left(s_{0}\right)=\Pi_{0} \gamma_{0}\left(s_{0}\right)
$$

where $\Pi_{0}$ is a $3 \times 3$ rotational matrix composed of three unit orthogonal vectors $\mathbf{r}_{X}, \mathbf{r}_{Y}$, and $\mathbf{r}_{Z}$ that are expressed in the inertial frame coordinates $O-x y z$ as shown in Figure 1, that is,

$$
\Pi_{0}=\left(\mathbf{r}_{X}, \mathbf{r}_{Y}, \mathbf{r}_{Z}\right)
$$

Since $\gamma_{0}\left(s_{0}\right)$ is parametrized by length parameter, $\gamma_{0}^{\prime}\left(s_{0}\right)=\mathrm{d} \gamma_{0}\left(s_{0}\right) / \mathrm{d}\left(s_{0}\right)$ must be expressed by the unit tangent vector $\mathbf{b}_{0}\left(s_{0}\right)$ at $P_{1}(s)$ lying on the tangent plane $T_{1}$. According to (a1) and (a2), it is possible to suppose that there exist the two unit normals $\mathbf{n}_{0}\left(s_{0}\right)$ and $\mathbf{n}_{1}\left(s_{1}\right)$ expressed in corresponding local coordinates $O_{m}-X Y Z$ and $O_{1}-X_{1} Y_{1} Z_{1}$, respectively (see Figure 2 ). Then, it is possible to certify that

$$
\overline{\mathbf{n}}_{0}=-\overline{\mathbf{n}}_{1}
$$

at $s_{0}=s_{1}=s$, where

$$
\overline{\mathbf{n}}_{0}=\Pi_{0} \mathbf{n}_{0}, \quad \overline{\mathbf{n}}_{1}=\Pi_{1} \mathbf{n}_{1},
$$




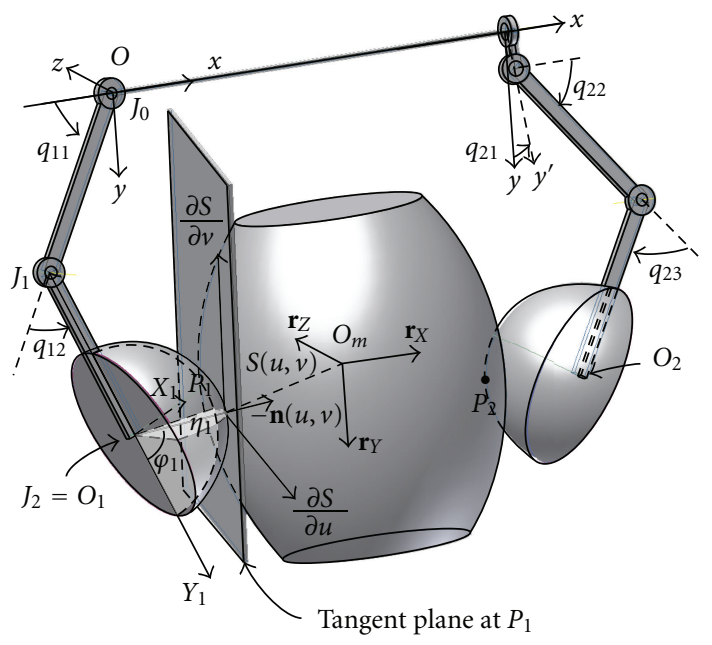

FIGURE 1: A pair of robot fingers grasping a 3D rigid object with smooth surfaces.

Frame coordinates

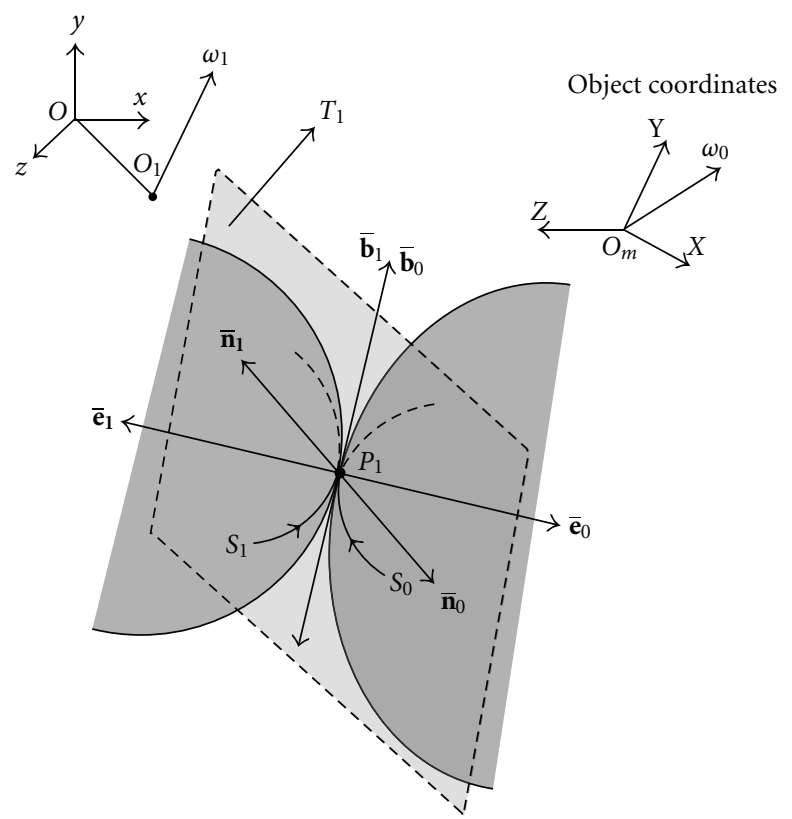

FIGURE 2: Definition of the moving frame coordinates system centering at the rolling contact point.

and $\Pi_{1}=\left(\mathbf{r}_{X 1}, \mathbf{r}_{Y 1}, \mathbf{r}_{Z 1}\right), \mathbf{r}_{X 1}$ denotes the unit vector of $X_{1}$-axis of $O_{1}-X_{1} Y_{1} Z_{1}$ expressed in the inertial frame coordinates, and $\mathbf{r}_{Y 1}$ and $\mathbf{r}_{Z 1}$ have a similar meaning.

In what follows, we denote vectors $\overline{\mathbf{n}}_{i}$ and $\overline{\mathbf{b}}_{i}$ for $i=0,1$ with upper bar when they are expressed in the inertial frame coordinates as seen in Figure 2. We also denote the derivative of $\Pi_{i}$ in time $t$ by $\dot{\Pi}_{i}$ and similarly the derivatives of $\mathbf{n}_{i}$ and $\mathbf{b}_{i}$ in $t$ by $\dot{\overline{\mathbf{n}}}_{i}$, and $\dot{\overline{\mathbf{b}}}_{i}$. If we assume that the instantaneous axis of angular velocity of the object through the mass center $O_{m}$ is denoted by $\bar{\omega}=\left(\omega_{x}, \omega_{y}, \omega_{z}\right)^{\mathrm{T}}$ in the frame coordinates, then the angular velocity vector $\boldsymbol{\omega}$ attached to the local coordinates $O_{m}-X Y Z$ can be defined in such a way that

$$
\overline{\boldsymbol{\omega}}=\Pi_{0}\left(\omega_{X}, \omega_{Y}, \omega_{Z}\right)^{\mathrm{T}}=\Pi_{0} \boldsymbol{\omega},
$$

where we define $\boldsymbol{\omega}=\left(\omega_{X}, \omega_{Y}, \omega_{Z}\right)^{\mathrm{T}}$. It is well known in the text books $[8-10]$ that

$$
\dot{\Pi}_{0}=\Pi_{0} \Omega_{0},
$$

where

$$
\Omega_{0}=\left(\begin{array}{ccc}
0 & -\omega_{Z} & \omega_{Y} \\
\omega_{Z} & 0 & -\omega_{X} \\
-\omega_{Y} & \omega_{X} & 0
\end{array}\right) .
$$

It is easy to check that, in the illustrative case of a spherical left hand fingertip shown in Figure 1, we have

$$
\dot{\Pi}_{1}=\Pi_{1} \Omega_{1}, \quad \Omega_{1}=\left(\begin{array}{ccc}
0 & \dot{p}_{1} & 0 \\
-\dot{p}_{1} & 0 & 0 \\
0 & 0 & 0
\end{array}\right),
$$

where $\dot{p}_{1}=\dot{q}_{11}+\dot{q}_{12}$ because both the rotational axes of joints $J_{1}$ and $J_{2}$ have the same direction in $z$-axis of the frame coordinates $O-x y z$.

Let us now interpret the first postulate (a1) in a mathematical form described by

$$
\mathbf{r}_{1}\left(q_{1}\right)+\Pi_{1} \gamma_{1}(s)=\mathbf{r}_{0}(\mathbf{x})+\Pi_{0} \gamma_{0}(s),
$$

where $\mathbf{r}_{1}\left(q_{1}\right)$ denotes the position vector of $O_{1}$ (the center of the left hand fingerend) expressed in terms of the frame coordinates and $q_{1}=\left(q_{11}, q_{12}\right)^{\mathrm{T}}$, and $\mathbf{r}_{0}(=\mathbf{x})$ does the position vector of $O_{m}$ (the object mass center) also expressed in the frame coordinates and $\mathbf{x}=(x, y, z)^{\mathrm{T}}$. Then, differentiation of (9) in $t$ yields

$$
\left(\dot{\mathbf{r}}_{1}-\dot{\mathbf{r}}_{0}\right)+\dot{\Pi}_{1} \gamma_{1}+\Pi_{1} \mathbf{b}_{1}\left(\frac{\mathrm{d} s_{1}}{\mathrm{~d} t}\right)=\dot{\Pi}_{0} \gamma_{0}+\Pi_{0} \mathbf{b}_{0}\left(\frac{\mathrm{d} s_{0}}{\mathrm{~d} t}\right) .
$$

If during rolling of the contact point the tangent vector $\overline{\mathbf{b}}_{1}$ of the fingerend has the same direction as that of $\overline{\mathbf{b}}_{0}$ (of the object), that is, if $\overline{\mathbf{b}}_{1}=\overline{\mathbf{b}}_{0}$, then on account of (a3), (10) reduces to

$$
\left(\dot{\mathbf{r}}_{1}-\dot{\mathbf{r}}_{0}\right)+\Pi_{1} \Omega_{1} \gamma_{1}-\Pi_{0} \Omega_{0} \gamma_{0}=0 .
$$

According to the previous paper, multiplication of the rotation matrix of the moving frame coordinates defined by $\Pi_{0} \Psi_{0}$ from the right yields

$$
\begin{aligned}
\left(R_{n 1}, R_{b 1}, R_{e 1}\right) \triangleq & \left(\dot{\mathbf{r}}_{1}-\dot{\mathbf{r}}_{0}\right)^{\mathrm{T}} \Pi_{0} \Psi_{0} \\
& -\boldsymbol{\omega}^{\mathrm{T}}\left(\gamma_{0} \times \Psi_{0}\right)+\boldsymbol{\omega}_{1}^{\mathrm{T}}\left(\gamma_{1} \times \Psi_{1}^{*}\right) \\
= & 0,
\end{aligned}
$$

where we define $\mathbf{e}_{0}=\mathbf{n}_{0} \times \mathbf{b}_{0}, \mathbf{e}_{1}=\mathbf{n}_{1} \times \mathbf{b}_{1}$, and

$$
\Psi_{0}=\left(\mathbf{n}_{0}, \mathbf{b}_{0}, \mathbf{e}_{0}\right), \quad \Psi_{1}^{*}=\left(-\mathbf{n}_{1}, \mathbf{b}_{1},-\mathbf{e}_{1}\right)
$$


(see Figure 2), and we use $\Pi_{0}=\Psi_{0}=\Pi_{1} \Psi^{*}$ and

$$
\gamma_{0} \times \Psi_{0}=\left(\gamma_{0} \times \mathbf{n}_{0}, \gamma_{0} \times \mathbf{b}_{0}, \gamma_{0} \times \mathbf{e}_{0}\right),
$$

and $\gamma_{1} \times \Psi_{1}^{*}$ has a similar meaning. Equation (13) means the three equalities $R_{n 1}=0, R_{b 1}=0$, and $R_{e 1}=0$ that constitute the set of three Pfaffian forms expressing the rolling contact constraint of zero-relative velocity. In the previous paper [7], it is shown that the Pfaffian forms of (13) are integrable with the integral forms

$$
\frac{\mathrm{d}}{\mathrm{d} t}\left(Q_{n 1}, Q_{b 1}, Q_{e 1}\right)=\left(R_{n 1}, R_{b 1}, R_{e 1}\right)=0,
$$

where

$$
\begin{gathered}
\left(Q_{n 1}, Q_{b 1}, Q_{e 1}\right)=\left(\mathbf{r}_{1}-\mathbf{r}_{0}\right)^{\mathrm{T}} \Pi_{0} \Psi_{0}+\gamma_{1}^{\mathrm{T}} \Psi_{1}^{*}-\gamma_{0}^{\mathrm{T}} \Psi_{0}, \\
\Psi_{1}=\left(\mathbf{n}_{1}, \mathbf{b}_{1}, \mathbf{e}_{1}\right)
\end{gathered}
$$

provided that $\overline{\mathbf{b}}_{0}=\overline{\mathbf{b}}_{1}$.

By virtue of the integrability of each Pfaffian form of rolling contact constraints, the Lagrangian of the system is written into

$$
\begin{aligned}
L= & K-M g y-\sum_{i=1,2} P_{i}\left(q_{i}\right) \\
& -\sum_{i=1,2}\left(f_{i} Q_{n i}+\lambda_{i} Q_{b i}+\eta_{i} Q_{e i}\right),
\end{aligned}
$$

where

$$
K=\sum_{i=1,2} \frac{1}{2} \dot{q}_{i}^{\mathrm{T}} G_{i}\left(q_{i}\right)+\frac{M}{2}\|\dot{\mathbf{x}}\|^{2}+\frac{1}{2} \boldsymbol{\omega}^{\mathrm{T}} H \boldsymbol{\omega} .
$$

In these equations, $M$ denotes the mass of the object, $H$, the inertia matrix of the object around its mass center, $G_{i}\left(q_{i}\right)$, the inertia matrix of finger $i, P_{i}$, the potential energy of finger $i$, $g$, the gravity constant, and $f_{i}, \lambda_{i}$, and $\eta_{i}$ express Lagrange multipliers corresponding to constraints

$$
\left(Q_{n 1}, Q_{b 1}, Q_{e 1}\right)=(0,0,0)
$$

and $\dot{\mathbf{x}}=\mathrm{d} \mathbf{r}(\mathbf{x}) / \mathrm{d} t$. Then, by applying the variational principle to $L$ described as

$$
\int_{t 0}^{t 1}\left\{\delta L+\sum_{i=1,2} u_{i}^{\mathrm{T}} \delta_{q i}\right\} \mathrm{d} t=0
$$

with control input $u_{i}$ at finger joints, it is possible to obtain the following set of Euler-Lagrange equations:

$$
\begin{aligned}
& M \ddot{\mathbf{x}}-\sum_{i=1,2}\left(f_{i} \overline{\mathbf{n}}_{0 i}+\lambda_{i} \overline{\mathbf{b}}_{0 i}+\eta_{i} \overline{\mathbf{e}}_{0 i}\right)+M g \mathbf{e}_{y}=0 \\
& H \dot{\boldsymbol{\omega}}+\boldsymbol{\omega} \times H \boldsymbol{\omega}-\sum_{i=1,2} \gamma_{0 i} \times\left(f_{i} \mathbf{n}_{0 i}+\lambda_{i} \mathbf{b}_{0 i}+\eta_{i} \mathbf{e}_{0 i}\right)=0 \\
& G_{i}\left(q_{i}\right) \ddot{q}_{i}+\left\{\frac{1}{2} \dot{G}_{i}\left(q_{i}\right)+S_{i}(q, \dot{q})\right\} \dot{q}_{i}+\frac{\partial P_{i}\left(q_{i}\right)}{\partial q_{i}} \\
& -J_{i}^{\mathrm{T}}\left(q_{i}\right)\left\{f_{i} \overline{\mathbf{n}}_{i}-\lambda_{i} \overline{\mathbf{b}}_{i}+\eta_{i} \overline{\mathbf{e}}_{i}\right\} \\
& -W_{i}^{\mathrm{T}}\left\{\gamma_{i} \times\left(f_{i} \mathbf{n}_{i}-\lambda_{i} \mathbf{b}_{i}+\eta_{i} \mathbf{e}_{i}\right)\right\}=u_{i}, \quad i=1,2
\end{aligned}
$$

where $\mathbf{e}_{y}=(0,1,0)^{\mathrm{T}}$, and the meaning of $W_{i}$ will be explained later. It should be noted that the sum of inner products of $\dot{\mathbf{x}}$ and (21), $\boldsymbol{\omega}$ and (22), and $\dot{q}_{i}$ and (23) for $i=1,2$ yields the energy relation

$$
\sum_{i=1,2} \dot{q}_{i}^{\mathrm{T}} u_{i}=\frac{\mathrm{d}}{\mathrm{d} t}\left\{K+M g y+\sum_{i=1,2} P_{i}\left(q_{i}\right)\right\} .
$$

\section{Necessary Conditions for Updating Moving Frame Coordinates}

In order to always keep the tangent vector $\mathbf{b}_{1}$ of the fingerend at the contact point $P_{1}$ to coincide with the tangent vector $\mathbf{b}_{0}$ of the object surface at the same common contact point, we first show that the following two equations should be satisfied necessarily:

$$
\begin{gathered}
\boldsymbol{\omega}_{0}^{\mathrm{T}} \mathbf{b}_{0}=\boldsymbol{\omega}_{1}^{\mathrm{T}} \mathbf{b}_{1}, \\
\left(\kappa_{n 0}+\kappa_{n 1}\right) \frac{\mathrm{d} s_{0}}{\mathrm{~d} t}=\boldsymbol{\omega}_{0}^{\mathrm{T}} \mathbf{e}_{0}+\boldsymbol{\omega}_{1}^{\mathrm{T}} \mathbf{e}_{1},
\end{gathered}
$$

as shown in detail in $\left(A_{1}\right)$ of Appendix A, where $\kappa_{n 0}$ denotes the normal curvature of the object surface at the contact point $P_{1}$, and $\kappa_{n 1}$ does that of the fingerend surface $S_{1}$ at $P_{1}$. Both the normal curvatures $\kappa_{n 0}$ and $\kappa_{n 1}$ should be determined in accordance with the geometric structure of their corresponding surfaces, once the direction of each locus of contact points, that is, $\mathbf{b}_{1}$ or $\mathbf{b}_{0}$, is given. Similarly, as shown in $\left(A_{2}\right)$ of Appendix $\mathrm{A}$, the conditions $\overline{\mathbf{b}}_{0}=\overline{\mathbf{b}}_{1}$ and $\dot{\overline{\mathbf{b}}}_{0}=\dot{\overline{\mathbf{b}}}_{1}$ imply

$$
\left(\boldsymbol{\omega}_{0}^{\mathrm{T}} \mathbf{n}_{0}+\boldsymbol{\omega}_{1}^{\mathrm{T}} \mathbf{n}_{1}\right)+\left(\kappa_{e 0}+\kappa_{e 1}\right) \frac{\mathrm{d} s_{0}}{\mathrm{~d} t}=0,
$$

where $\kappa_{e 0}$ denotes the geodesic curvature of the object surface at $P_{1}$, and $\kappa_{e 1}$ has a similar meaning. This equation does not determine each $\kappa_{e 0}$ or $\kappa_{e 1}$ individually. Therefore, let us try to differentiate $\overline{\mathbf{e}}_{0}$ and $\overline{\mathbf{e}}_{1}$ in $t$. However, as shown in $\left(A_{3}\right)$ of Appendix A, we rederive only (25) and (26).

We are now in a position to find a necessary condition for maintaining the equality of (25) for the time being. To this end, it is important to see that the time rate of the equality (25) reduces to

$$
\begin{aligned}
& \left\{\left(\boldsymbol{\omega}_{0}^{\mathrm{T}} \mathbf{e}_{0}\right) \kappa_{e 0}-\left(\boldsymbol{\omega}_{1}^{\mathrm{T}} \mathbf{e}_{1}\right) \kappa_{e 1}\right\} \frac{\mathrm{d} s_{0}}{\mathrm{~d} t} \\
& =\dot{\boldsymbol{\omega}}_{1}^{\mathrm{T}} \mathbf{b}_{1}-\dot{\boldsymbol{\omega}}_{0}^{\mathrm{T}} \mathbf{b}_{0}+\left(-\kappa_{n 0} \boldsymbol{\omega}_{0}^{\mathrm{T}} \mathbf{n}_{0}+\kappa_{n 1} \boldsymbol{\omega}_{1}^{\mathrm{T}} \mathbf{n}_{1}\right),
\end{aligned}
$$

as shown in $\left(A_{4}\right)$ of Appendix A. Then, this equation together with (27) leads to

$$
\left(\begin{array}{cc}
1 & 1 \\
\boldsymbol{\omega}_{0}^{\mathrm{T}} \mathbf{e}_{0} & -\boldsymbol{\omega}_{1}^{\mathrm{T}} \mathbf{e}_{1}
\end{array}\right)\left(\begin{array}{c}
\kappa_{e 0} \\
\kappa_{e 1}
\end{array}\right) \frac{\mathrm{d} s_{0}}{\mathrm{~d} t}=\left(\begin{array}{c}
-\left(\boldsymbol{\omega}_{0}^{\mathrm{T}} \mathbf{n}_{0}+\boldsymbol{\omega}_{1}^{\mathrm{T}} \mathbf{n}_{1}\right) \\
\xi_{1}
\end{array}\right),
$$


where

$$
\xi_{1} \triangleq\left(-\kappa_{n 0} \boldsymbol{\omega}_{0}^{\mathrm{T}} \mathbf{n}_{0}+\kappa_{n 1} \boldsymbol{\omega}_{1}^{\mathrm{T}} \mathbf{n}_{1}\right) \frac{\mathrm{d} s_{0}}{\mathrm{~d} t}+\dot{\boldsymbol{\omega}}_{1}^{\mathrm{T}} \mathbf{b}_{1}-\dot{\boldsymbol{\omega}}_{0}^{\mathrm{T}} \mathbf{b}_{0} .
$$

Thus, it is possible to determine each geodesic curvature individually by inverting the coefficient $2 \times 2$ matrix of (29) in the following way:

$$
\begin{aligned}
\kappa_{e 0} \frac{\mathrm{d} s_{0}}{\mathrm{~d} t}=\frac{-\boldsymbol{\omega}_{1}^{\mathrm{T}} \mathbf{e}_{1}\left(\boldsymbol{\omega}_{0}^{\mathrm{T}} \mathbf{n}_{0}+\boldsymbol{\omega}_{1}^{\mathrm{T}} \mathbf{n}_{1}\right)+\xi_{1}}{\boldsymbol{\omega}_{1}^{\mathrm{T}} \mathbf{e}_{1}+\boldsymbol{\omega}_{0}^{\mathrm{T}} \mathbf{e}_{0}}, \\
\kappa_{e 1} \frac{\mathrm{d} s_{1}}{\mathrm{~d} t}=\frac{-\boldsymbol{\omega}_{0}^{\mathrm{T}} \mathbf{e}_{0}\left(\boldsymbol{\omega}_{0}^{\mathrm{T}} \mathbf{n}_{0}+\boldsymbol{\omega}_{1}^{\mathrm{T}} \mathbf{n}_{1}\right)-\xi_{1}}{\boldsymbol{\omega}_{1}^{\mathrm{T}} \mathbf{e}_{1}+\boldsymbol{\omega}_{0}^{\mathrm{T}} \mathbf{e}_{0}} .
\end{aligned}
$$

Finally, it is possible to see that the moving frames denoted by $\Psi_{0}$ and $\Psi_{1}$ should satisfy the Frenet-Serre equations

$$
\begin{aligned}
& \frac{\mathrm{d}}{\mathrm{d} t} \Psi_{0}=\Psi_{0}\left(\begin{array}{ccc}
0 & \kappa_{n 0} & 0 \\
-\kappa_{n 0} & 0 & -\kappa_{e 0} \\
0 & \kappa_{e 0} & 0
\end{array}\right) \frac{\mathrm{d} s_{0}}{\mathrm{~d} t}, \\
& \frac{\mathrm{d}}{\mathrm{d} t} \Psi_{1}=\Psi_{1}\left(\begin{array}{ccc}
0 & \kappa_{n 1} & 0 \\
-\kappa_{n 1} & 0 & -\kappa_{e 1} \\
0 & \kappa_{e 1} & 0
\end{array}\right) \frac{\mathrm{d} s_{0}}{\mathrm{~d} t}
\end{aligned}
$$

\section{Sufficient Conditions for Updating the Moving Frame Coordinates}

In the Frenet-Serre equation of (31), the coefficient $\kappa_{n 0}$ called the normal curvature is determined by the geometric shape of the object surface at point $P_{1}$ denoted by $\gamma_{0}\left(s_{0}\right)$ in the local coordinates $O_{m}-X Y Z$, and the other normal curvature $\kappa_{n 1}$ in (32) is also determined similarly. The geodesic curvatures $\kappa_{e 0}$ and $\kappa_{e 1}$ are determined via the instantaneous motion of rolling contact, so that they satisfy (27) and (28). We now show under the postulates (a1) to (a3) that if $\kappa_{e 0}$ and $\kappa_{e 1}$ are determined by (31) and (32), respectively, and the tangent vectors $\overline{\mathbf{b}}_{0}(0)$ and $\overline{\mathbf{b}}_{1}(0)$ of the moving frame coordinates at the initial time are chosen to coincide with each other and at the same time to satisfy (25) at $t=0$, then for any $t>0$, it follows that

$$
\begin{gathered}
\frac{\mathrm{d}}{\mathrm{d} t} \overline{\mathbf{b}}_{1}=\frac{\mathrm{d}}{\mathrm{d} t} \overline{\mathbf{b}}_{0}, \quad \frac{\mathrm{d}}{\mathrm{d} t} \overline{\mathbf{e}}_{1}=-\frac{\mathrm{d}}{\mathrm{d} t} \overline{\mathbf{e}}_{0}, \\
\overline{\mathbf{b}}_{1}=\overline{\mathbf{b}}_{0}, \quad \overline{\mathbf{e}}_{1}=\overline{\mathbf{e}}_{0} .
\end{gathered}
$$

To prove this, first note again that the postulates (a1) and (a2) imply

$$
\overline{\mathbf{n}}_{0}=-\overline{\mathbf{n}}_{1}, \quad \frac{\mathrm{d}}{\mathrm{d} t} \overline{\mathbf{n}}_{0}=-\frac{\mathrm{d}}{\mathrm{d} t} \overline{\mathbf{n}}_{1} .
$$

Next, note that the sum of (31) and (32) implies (27). At this stage, suppose that $\overline{\mathbf{b}}_{1}$ is not coincident with $\overline{\mathbf{b}}_{0}$ at some $t>0$ though both $\overline{\mathbf{b}}_{1}$ and $\overline{\mathbf{b}}_{0}$ are lying on the same tangent plane.
In other words, suppose that $\overline{\mathbf{b}}_{1}^{\mathrm{T}} \overline{\mathbf{b}}_{0}=\cos \theta$ and $\theta(t)$ stands for $\theta\left(t, s_{1}(t), s_{0}(t)\right)$, then, as shown in Appendix B, we have

$$
\dot{\theta}=\boldsymbol{\omega}_{1}^{\mathrm{T}} \mathbf{n}_{1}+\boldsymbol{\omega}_{0}^{\mathrm{T}} \mathbf{n}_{0}+\left(\kappa_{e 0}+\kappa_{e 1}\right) \frac{\mathrm{d} s_{0}}{\mathrm{~d} t} .
$$

Further, as discussed in $\left(B_{3}\right)$ of Appendix B, geodesic curvatures $\kappa_{e 0}$ and $\kappa_{e 1}$ should be defined as

$$
\kappa_{e 0}=\frac{\partial \theta}{\partial s_{0}}, \quad \kappa_{e 1}=\frac{\partial \theta}{\partial s_{1}} .
$$

It is important to remark that (36) was first derived by Montana [2] as a nonholonomic constraint of rolling. Equation (36) can be interpreted by Murray et al. [1] as the nonholonomic constraint that governs the rotating motion of one tangent plane to the fingerend relative to another tangent plane of the object surface caused by relative "twisting" between the axis of normal $\overline{\mathbf{n}}_{1}$ and that of $\overline{\mathbf{n}}_{0}$. Nevertheless, it is further important to note that if $\kappa_{e 0}$ and $\kappa_{e 1}$ are set as shown in (31) and (32), respectively, then the right hand side of (36) becomes zero due to (27). That is, (31) and (32) imply $\dot{\theta}=0$. Therefore, if at the initial time $\overline{\mathbf{b}}_{1}(0)=\overline{\mathbf{b}}_{0}(0)$, then the setting of (31) and (32) for geodesic curvatures $\kappa_{e 1}$ and $\kappa_{e 0}$ leads to $\overline{\mathbf{b}}_{1}(t)=\overline{\mathbf{b}}_{0}(t)$ for $t>0$ as far as $\boldsymbol{\omega}_{1}^{\mathrm{T}} \mathbf{e}_{1}+\boldsymbol{\omega}_{0}^{\mathrm{T}} \mathbf{e}_{0} \neq 0$. Since $\overline{\mathbf{n}}_{1}=-\overline{\mathbf{n}}_{0}$ as far as the contact is maintained, the equality $\overline{\mathbf{b}}_{1}(t)=\overline{\mathbf{b}}_{0}(t)$ implies $\overline{\mathbf{e}}_{1}(t)=-\overline{\mathbf{e}}_{0}(t)$ for $t \geq 0$, then, from $\left(A_{1}\right)$ of Appendix A, (25) and (26) follow. At the same time, from (26) and (27), it follows that $\mathrm{d} \overline{\mathbf{b}}_{1} / \mathrm{d} t=\mathrm{d} \overline{\mathbf{b}}_{0} / \mathrm{d} t$ in view of the first two equations of $\left(A_{2}\right)$ of Appendix A. Thus, it is concluded that all equalities in (34) follow.

\section{A Numerically Integrable Set of Differential Equations Under Rolling Constraints}

It is now possible to show a set of all the differential equations of motion of the fingers-object system under rolling contact constraints. In what follows, we use the suffix " $0 i$ " for expressing variables on quantities of the object at contact point $P_{i}$ of the $i$-th finger and the suffix $i$ for those of the fingerend surface of finger $i$ at $P_{i}$. For convenience, we use $\boldsymbol{\omega}$ instead of $\boldsymbol{\omega}_{0}$. In the following, we give the set of Euler-Lagrange equations, first-order equations of rotation matrices $\Pi_{0}$ and $\Pi_{i}$, update equations of length parameters, and Frenet-Serre equations for updating moving frame coordinates at contact points

$$
\begin{gathered}
M \ddot{\mathbf{x}}-\sum_{i} \Pi_{0} \Psi_{0 i} \boldsymbol{\lambda}_{i}-M g \mathbf{e}_{y}=0, \\
H \dot{\boldsymbol{\omega}}+\boldsymbol{\omega} \times H \boldsymbol{\omega}-\sum_{i} \gamma_{0 i} \times \Psi_{0 i} \boldsymbol{\lambda}_{i}=0, \\
G_{i} \ddot{q}_{i}+\left\{\frac{1}{2} \dot{G}_{i}+S_{i}\right\} \dot{q}_{i}+\frac{\partial P_{i}}{\partial q_{i}} \\
+\left\{J_{i}^{\mathrm{T}} \Pi_{i}+W_{i}^{\mathrm{T}} \gamma_{i} \times\right\} \Psi_{i}^{*} \boldsymbol{\lambda}_{i}=u_{i},
\end{gathered}
$$




$$
\begin{gathered}
\frac{\mathrm{d}}{\mathrm{d} t} \Pi_{0}=\Pi_{0} \Omega_{0}, \quad \frac{\mathrm{d}}{\mathrm{d} t} \Pi_{i}=\Pi_{i} \Omega_{i}, \\
\frac{\mathrm{d}}{\mathrm{d} t} s_{i}=\frac{\boldsymbol{\omega}^{\mathrm{T}} \mathbf{e}_{0 i}+\boldsymbol{\omega}_{i}^{\mathrm{T}} \mathbf{e}_{i}}{\kappa_{n 0 i}+\kappa_{n i}}, \\
\frac{\mathrm{d}}{\mathrm{d} t} \Psi_{0 i}=\Psi_{0 i} K_{0 i} \frac{\mathrm{d} s_{i}}{\mathrm{~d} t}, \quad \frac{\mathrm{d}}{\mathrm{d} t} \Psi_{i}=\Psi_{i} K_{i} \frac{\mathrm{d} s_{i}}{\mathrm{~d} t},
\end{gathered}
$$

where

$$
\begin{gathered}
K_{0 i}=\left(\begin{array}{ccc}
0 & \kappa_{n 0 i} & 0 \\
-\kappa_{n 0 i} & 0 & -\kappa_{e 0 i} \\
0 & \kappa_{e 0 i} & 0
\end{array}\right), \\
K_{i}=\left(\begin{array}{ccc}
0 & \kappa_{n i} & 0 \\
-\kappa_{n i} & 0 & -\kappa_{e i} \\
0 & \kappa_{e i} & 0
\end{array}\right), \\
\Psi_{0 i}=\left(\mathbf{n}_{0 i}, \mathbf{b}_{0 i}, \mathbf{e}_{0 i}\right), \\
\lambda_{i}=\left(f_{i}, \lambda_{i}, \eta_{i}\right)^{\mathrm{T}},
\end{gathered}
$$

and $\gamma_{0 i}$ denotes the contact point $P_{i}$ on the object surface expressed by the object local coordinates $O_{m}-X Y Z$, and $\gamma_{i}$ does that of $P_{i}$ on the fingerend surface of finger $i$ expressed by the fingerend local coordinates $O_{i}-X_{i} Y_{i} Z_{i}$. In $\left(E_{i}\right), W_{i}$ is an $m \times 3$ matrix depending on $q_{i}$, where $m$ denotes the degrees of freedom. In the case of a pair of robot fingers depicted in Figure 1, it is obvious to see that

$$
W_{1}=\left(\begin{array}{ll}
0 & 0 \\
0 & 0 \\
1 & 1
\end{array}\right), \quad W_{2}=\left(\begin{array}{ccc}
1 & 0 & 0 \\
0 & \sin q_{21} & \sin q_{21} \\
0 & \cos q_{21} & \cos q_{21}
\end{array}\right) .
$$

It should be remarked again that $\left(E_{f s}\right)$ expresses a set of Frenet-Serre equations for determining each moving frame coordinates at contact point $P_{i}$, and then the geodesic curvatures $\kappa_{e 0 i}$ and $\kappa_{e i}$ are determined in the same manner as shown in (31) and (32). Further, computation of $\dot{\boldsymbol{\omega}}_{i}$ and $\dot{\boldsymbol{\omega}}$ appearing in (31) and (32) through $\xi_{i}$ defined by (30) can be executed simultaneously via numerical integration of $\left(E_{\omega}\right)$ and $\left(E_{i}\right)$. In practice, it is possible to compute $\dot{\boldsymbol{\omega}}_{i}$ by

$$
\dot{\omega}=H^{-1}\left\{-\boldsymbol{\omega} \times H \boldsymbol{\omega}+\sum_{i} \gamma_{0 i} \times \Psi_{0 i} \lambda_{i}\right\} .
$$

Analogously, it is possible to compute $\dot{\boldsymbol{\omega}}_{i}$ since $\boldsymbol{\omega}_{i}$ must be expressed by a function form of $V\left(q_{i}\right) \dot{q}_{i}$, and $\ddot{q}_{i}$ can be calculated by multiplying $\left(E_{i}\right)$ by $G_{i}^{-1}\left(q_{i}\right)$ from the left.

\section{Conclusions}

A computational model of dynamics of 3D object grasping and manipulation under rolling contact constraints by means of multiple multijoint robot fingers with smooth fingerend surfaces is derived on the basis of the postulates of pure rolling contact constraint. The postulates are summarized: (1) at the contact point, the fingerend and object surfaces share a common tangent plane with each normal with opposite direction and (2) the path length of contact points running on the fingerend is coincident with that running on the object surface. The postulates are adopted by referring to Nomizu's work [11] in which it is assumed that any relative twist motion does not arise. The proposed model is composed of a set of 2nd-order Euler-Lagrange equations derived by using the moving frame coordinates and 1st-order Frenet-Serre equations together with 1storder differential equations governing update laws of length parameters and rotational motions of the local coordinates. The nonholonomic constraint arising from possible relative twist of the two normal axes at the contact point is resolved into determination of the geodesic curvatures of the fingerend and object surfaces. This leads to a conclusion that the whole set of simultaneous differential equations with constraints are numerically integrable (as a preliminary result of numerical simulation, see [12]).

\section{Appendices}

\section{A. Necessary Conditions}

$\left(A_{1}\right)$ Note that $\overline{\mathbf{n}}_{1}=-\overline{\mathbf{n}}_{0}$ and $(\mathrm{d} / \mathrm{d} t) \overline{\mathbf{n}}_{1}=-(\mathrm{d} / \mathrm{d} t) \overline{\mathbf{n}}_{0}$,

$$
\begin{aligned}
\frac{\mathrm{d}}{\mathrm{d} t} \overline{\mathbf{n}}_{0} & =\dot{\Pi}_{0} \mathbf{n}_{0}+\Pi_{0} \dot{\mathbf{n}}_{0} \\
& =\Pi_{0} \Omega_{0} \mathbf{n}_{0}+\Pi_{0}\left(\frac{\partial \mathbf{n}_{0}}{\partial s_{0}}\right) \frac{\mathrm{d} s_{0}}{\mathrm{~d} t} \\
& =\Pi_{0}\left\{\boldsymbol{\omega}_{0} \times\left(\mathbf{b}_{0} \times \mathbf{e}_{0}\right)\right\}-\kappa_{n 0} \Pi_{0} \mathbf{b}_{0} \frac{\mathrm{d} s_{0}}{\mathrm{~d} t} \\
& =\left(\boldsymbol{\omega}_{0}^{\mathrm{T}} \mathbf{e}_{0}-\kappa_{n 0} \frac{\mathrm{d} s_{0}}{\mathrm{~d} t}\right) \overline{\mathbf{b}}_{0}-\left(\boldsymbol{\omega}_{0}^{\mathrm{T}} \mathbf{b}_{0}\right) \overline{\mathbf{e}}_{0},
\end{aligned}
$$

$\frac{\mathrm{d}}{\mathrm{d} t} \overline{\mathbf{n}}_{1}=\left(\boldsymbol{\omega}_{1}^{\mathrm{T}} \mathbf{e}_{1}-\kappa_{n_{1}} \frac{\mathrm{d} s_{1}}{\mathrm{~d} t}\right) \overline{\mathbf{b}}_{1}-\left(\boldsymbol{\omega}_{1}^{\mathrm{T}} \mathbf{b}_{1}\right) \overline{\mathbf{e}}_{1}$.

If $\overline{\mathbf{b}}_{0}=\overline{\mathbf{b}}_{1}$ and $\overline{\mathbf{e}}_{0}=-\overline{\mathbf{e}}_{1}$, then it follows that

$$
\begin{gathered}
\boldsymbol{\omega}_{0}^{\mathrm{T}} \mathbf{b}_{0}=\boldsymbol{\omega}_{1}^{\mathrm{T}} \mathbf{b}_{1}, \\
\left(\kappa_{n 0}+\kappa_{n 1}\right) \frac{\mathrm{d} s_{0}}{\mathrm{~d} t}=\boldsymbol{\omega}_{0}^{\mathrm{T}} \mathbf{e}_{0}+\boldsymbol{\omega}_{1}^{\mathrm{T}} \mathbf{e}_{1} .
\end{gathered}
$$

$\left(A_{2}\right)$ Similarly, it follows that

$$
\begin{aligned}
\frac{\mathrm{d}}{\mathrm{d} t} \overline{\mathbf{b}}_{0}= & \dot{\Pi}_{0} \mathbf{b}_{0}+\Pi_{0} \dot{\mathbf{b}}_{0} \\
= & \Pi_{0}\left\{\left(\boldsymbol{\omega}_{0}^{\mathrm{T}} \mathbf{n}_{0}\right) \mathbf{e}_{0}-\left(\boldsymbol{\omega}_{0}^{\mathrm{T}} \mathbf{e}_{0}\right) \mathbf{n}_{0}\right\} \\
& +\Pi_{0}\left(\kappa_{n 0} \mathbf{n}_{0}+\kappa_{e 0} \mathbf{e}_{0}\right) \frac{\mathrm{d} s_{0}}{\mathrm{~d} t} \\
= & \left(\boldsymbol{\omega}_{0}^{\mathrm{T}} \mathbf{n}_{0}+\kappa_{e 0} \frac{\mathrm{d} s_{0}}{\mathrm{~d} t}\right) \overline{\mathbf{e}}_{0} \\
& -\left(\boldsymbol{\omega}_{0}^{\mathrm{T}} \mathbf{e}_{0}+\kappa_{n 0} \frac{\mathrm{d} s_{0}}{\mathrm{~d} t}\right) \overline{\mathbf{n}}_{0}
\end{aligned}
$$




$$
\begin{aligned}
\frac{\mathrm{d}}{\mathrm{d} t} \overline{\mathbf{b}}_{1}= & \left(\boldsymbol{\omega}_{1}^{\mathrm{T}} \mathbf{n}_{1}+\kappa_{e 1} \frac{\mathrm{d} s_{1}}{\mathrm{~d} t}\right) \overline{\mathbf{e}}_{1} \\
& -\left(\boldsymbol{\omega}_{1}^{\mathrm{T}} \mathbf{e}_{1}+\kappa_{n 1} \frac{\mathrm{d} s_{1}}{\mathrm{~d} t}\right) \overline{\mathbf{n}}_{1} .
\end{aligned}
$$

These two equations imply $\left(E_{i}\right)$ and

$$
\left(\boldsymbol{\omega}_{0}^{\mathrm{T}} \mathbf{n}_{0}+\boldsymbol{\omega}_{1}^{\mathrm{T}} \mathbf{n}_{1}\right)+\left(\kappa_{e 0}+\kappa_{e 1}\right) \frac{\mathrm{d} s_{0}}{\mathrm{~d} t}=0
$$

if $\overline{\mathbf{b}}_{0}=\overline{\mathbf{b}}_{1}$ and $(\mathrm{d} / \mathrm{d} t) \overline{\mathbf{b}}_{0}=(\mathrm{d} / \mathrm{d} t) \overline{\mathbf{b}}_{1}$.

$\left(A_{3}\right)$ Similarly, it follows that

$$
\begin{gathered}
\frac{\mathrm{d}}{\mathrm{d} t} \overline{\mathbf{e}}_{0}=\left(\boldsymbol{\omega}_{0}^{\mathrm{T}} \mathbf{b}_{0}\right) \overline{\mathbf{n}}_{0}-\left(\boldsymbol{\omega}_{0}^{\mathrm{T}} \mathbf{n}_{0}+\kappa_{e 0} \frac{\mathrm{d} s_{0}}{\mathrm{~d} t}\right) \overline{\mathbf{b}}_{0}, \\
\frac{\mathrm{d}}{\mathrm{d} t} \overline{\mathbf{e}}_{1}=\left(\boldsymbol{\omega}_{1}^{\mathrm{T}} \mathbf{b}_{1}\right) \overline{\mathbf{n}}_{1}-\left(\boldsymbol{\omega}_{0}^{\mathrm{T}} \mathbf{n}_{1}+\kappa_{e 1} \frac{\mathrm{d} s_{1}}{\mathrm{~d} t}\right) \overline{\mathbf{b}}_{1} .
\end{gathered}
$$

These two equations imply $\left(E_{\omega}\right)$ and $\left(E_{s}\right)$ if $\overline{\mathbf{e}}_{0}=-\overline{\mathbf{e}}_{1}$, $\overline{\mathbf{b}}_{0}=\overline{\mathbf{b}}_{1}$, and $(\mathrm{d} / \mathrm{d} t) \overline{\mathbf{e}}_{0}=-(\mathrm{d} / \mathrm{d} t) \overline{\mathbf{e}}_{1}$.

$\left(A_{4}\right)$ Time rate of $\left(E_{\omega}\right)$ reduces to

$$
\dot{\boldsymbol{\omega}}_{0}^{\mathrm{T}} \mathbf{b}_{0}+\boldsymbol{\omega}_{0}^{\mathrm{T}} \dot{\mathbf{b}}_{0}=\dot{\boldsymbol{\omega}}_{1}^{\mathrm{T}} \mathbf{b}_{1}+\boldsymbol{\omega}_{1}^{\mathrm{T}} \dot{\mathbf{b}}_{1}
$$

Since $\dot{\mathbf{b}}_{i}=\left(\kappa_{n i} \mathbf{n}_{i}+\kappa_{e i} \mathbf{e}_{i}\right)\left(\mathrm{d} s_{0} / \mathrm{d} t\right)$ for $i=0,1$, the above equality reduces to

$$
\begin{aligned}
\left\{\left(\boldsymbol{\omega}_{0}^{\mathrm{T}} \mathbf{e}_{0}\right) \kappa_{e 0}-\left(\boldsymbol{\omega}_{1}^{\mathrm{T}} \mathbf{e}_{1}\right) \kappa_{e 1}\right\} \frac{\mathrm{d} s_{0}}{\mathrm{~d} t} & \left(-\kappa_{n 0} \boldsymbol{\omega}_{0}^{\mathrm{T}} \mathbf{n}_{0}+\kappa_{n 1} \boldsymbol{\omega}_{1}^{\mathrm{T}} \mathbf{n}_{1}\right) \frac{\mathrm{d} s_{0}}{\mathrm{~d} t} \\
& +\dot{\boldsymbol{\omega}}_{1}^{\mathrm{T}} \mathbf{b}_{1}-\dot{\boldsymbol{\omega}}_{0}^{\mathrm{T}} \mathbf{b}_{0} \\
\triangleq & \xi_{1}\left(\dot{\boldsymbol{\omega}}_{1}, \dot{\boldsymbol{\omega}}_{0}\right) .
\end{aligned}
$$

\section{B. Preliminary Remarks on Geodesic Curvature}

$\overline{\mathbf{b}}_{0 i}=\Pi_{0} \mathbf{b}_{0 i} \quad$ (tangent vectors on object surface $\left.i\right)$,

$\overline{\mathbf{n}}_{0 i}=\Pi_{0} \mathbf{n}_{0 i} \quad$ (normal vectors on object surface $i$ ),

$\overline{\mathbf{b}}_{i}=\Pi_{i} \mathbf{b}_{i} \quad$ (tangent vectors on fingerend surface $\left.i\right)$,

$\overline{\mathbf{n}}_{i}=\prod_{i} \mathbf{n}_{i} \quad($ normal vectors on fingerend surface $i)$,

both $\Omega_{i}$ and $\Omega_{0}$ are skew symmetric.

$\left(B_{1}\right)$ Derivation of $\partial \theta / \partial t$ where $\theta\left(t, s_{i}(t), s_{i 0}(t)\right)$.

$$
\begin{aligned}
\text { If } \overline{\mathbf{b}}_{1}^{\mathrm{T}} \overline{\mathbf{b}}_{0}= & \cos \theta \text {, then } \\
\mathbf{b}_{1}^{\mathrm{T}} \Pi_{1}^{\mathrm{T}} \Pi_{0} \mathbf{e}_{0}= & \cos \left(\frac{\pi}{2}+\theta\right)=-\sin \theta \\
-\frac{\partial \theta}{\partial t} \cos \theta= & \frac{\theta}{\partial t}\left(\mathbf{b}_{1}^{\mathrm{T}} \Pi_{1}^{\mathrm{T}} \Pi_{0} \mathbf{e}_{0}\right) \\
= & \mathbf{b}_{1}^{\mathrm{T}} \dot{\Pi}_{1}^{\mathrm{T}} \Pi_{0} \mathbf{e}_{0}+\mathbf{b}_{1}^{\mathrm{T}} \Pi_{1}^{\mathrm{T}} \dot{\Pi}_{0} \mathbf{e}_{0} \\
= & \mathbf{b}_{1}^{\mathrm{T}} \Omega_{1}^{\mathrm{T}} \Pi_{1}^{\mathrm{T}} \Pi_{0} \mathbf{e}_{0}+\mathbf{b}_{1}^{\mathrm{T}} \Pi_{1}^{\mathrm{T}} \Pi_{0} \Omega_{0} \mathbf{e}_{0} \\
= & \left\{\Omega_{1}\left(\mathbf{e}_{1} \times \mathbf{n}_{1}\right)\right\}^{\mathrm{T}} \Pi_{1}^{\mathrm{T}} \Pi_{0} \mathbf{e}_{0} \\
& +\mathbf{b}_{1}^{\mathrm{T}} \Pi_{1}^{\mathrm{T}} \Pi_{0} \Omega_{0}\left(\mathbf{n}_{0} \times \mathbf{b}_{0}\right) \\
= & \left\{\left(\boldsymbol{\omega}_{1}^{\mathrm{T}} \mathbf{n}_{1}\right) \mathbf{e}_{1}-\left(\boldsymbol{\omega}_{1}^{\mathrm{T}} \mathbf{e}_{1}\right) \mathbf{n}_{1}\right\} \Pi_{1}^{\mathrm{T}} \Pi_{0} \mathbf{e}_{0} \\
& +\mathbf{b}_{1}^{\mathrm{T}} \Pi_{1}^{\mathrm{T}} \Pi_{0}\left\{\left(\boldsymbol{\omega}_{0}^{\mathrm{T}} \mathbf{b}_{0}\right) \mathbf{n}_{0}-\left(\boldsymbol{\omega}_{0}^{\mathrm{T}} \mathbf{n}_{0}\right) \mathbf{b}_{0}\right\} \\
= & \left(\boldsymbol{\omega}_{1}^{\mathrm{T}} \mathbf{n}_{1}\right) \mathbf{e}_{1}^{\mathrm{T}} \Pi_{1}^{\mathrm{T}} \Pi_{0} \mathbf{e}_{0}-\mathbf{b}_{1}^{\mathrm{T}} \Pi_{1} \Pi_{0} \mathbf{b}_{0}\left(\boldsymbol{\omega}_{0}^{\mathrm{T}} \mathbf{n}_{0}\right) \\
= & -\left(\boldsymbol{\omega}_{1}^{\mathrm{T}} \mathbf{n}_{1}+\boldsymbol{\omega}_{0}^{\mathrm{T}} \mathbf{n}_{0}\right) \cos \theta, \\
\frac{\partial \theta}{\partial t}= & \boldsymbol{\omega}_{1}^{\mathrm{T}} \mathbf{n}_{1}+\boldsymbol{\omega}_{0}^{\mathrm{T}} \mathbf{n}_{0} .
\end{aligned}
$$

$\left(B_{2}\right)$ With derivation of $\dot{\theta}$,

$$
\begin{aligned}
\mathbf{b}_{1}^{\mathrm{T}} \Pi_{1}^{\mathrm{T}} \Pi_{0} \mathbf{e}_{0}= & -\sin \theta \\
-\dot{\theta} \cos \theta= & -\left(\boldsymbol{\omega}_{1}^{\mathrm{T}} \mathbf{n}_{1}+\boldsymbol{\omega}_{0}^{\mathrm{T}} \mathbf{n}_{0}\right) \cos \theta \\
& +\mathbf{b}_{1}^{\mathrm{T}} \Pi_{1}^{\mathrm{T}} \Pi_{0}\left(-\kappa_{\mathrm{e} 0} \mathbf{b}_{0}\right) \frac{\mathrm{d} s_{0}}{\mathrm{~d} t} \\
& +\left(\kappa_{n 1} \mathbf{n}_{1}+\kappa_{e 1} \mathbf{e}_{1}\right)^{\mathrm{T}} \Pi_{1}^{\mathrm{T}} \Pi_{0} \mathbf{e}_{0} \frac{\mathrm{d} s_{1}}{\mathrm{~d} t} \\
= & -\left(\boldsymbol{\omega}_{1}^{\mathrm{T}} \mathbf{n}_{1}+\boldsymbol{\omega}_{0}^{\mathrm{T}} \mathbf{n}_{0}\right) \cos \theta \\
& -\kappa_{e 0} \cos \theta \frac{\mathrm{d} s_{0}}{\mathrm{~d} t}-\kappa_{e 1} \cos \theta \frac{\mathrm{d} s_{1}}{\mathrm{~d} t}, \\
\dot{\theta}= & \boldsymbol{\omega}_{1}^{\mathrm{T}} \mathbf{n}_{1}+\boldsymbol{\omega}_{0}^{\mathrm{T}} \mathbf{n}_{0}+\left(\kappa_{e 0}+\kappa_{e 1}\right) \frac{\mathrm{d} s_{1}}{\mathrm{~d} t} .
\end{aligned}
$$

$\left(B_{3}\right)$ With derivation of $\kappa_{e 0}$ and $\kappa_{e 1}$,

$$
\begin{aligned}
\mathbf{b}_{1}^{\mathrm{T}} \Pi_{1}^{\mathrm{T}} \Pi_{0} \mathbf{e}_{0} & =-\sin \theta, \\
\frac{\partial}{\partial s_{0}} \mathbf{b}_{1}^{\mathrm{T}} \Pi_{1}^{\mathrm{T}} \Pi_{0} \mathbf{e}_{0} & =-\frac{\partial \theta}{\partial s_{0}} \cos \theta \\
\mathbf{b}_{1}^{\mathrm{T}} \Pi_{1}^{\mathrm{T}} \Pi_{0}\left(-\kappa_{e 0}\right) \mathbf{b}_{0} & =-\frac{\partial \theta}{\partial s_{0}} \cos \theta \\
-\kappa_{e 0} \cos \theta & =-\frac{\partial \theta}{\partial s_{0}} \cos \theta, \\
\kappa_{e 0} & =\frac{\partial \theta}{\partial s_{0}} \\
\kappa_{e 1} & =\frac{\partial \theta}{\partial s_{1}} .
\end{aligned}
$$




\section{References}

[1] R. M. Murray, Z. Li, and S. S. Sastry, A Mathematical Introduction to Robotic Manipulation, CRC Press, Boca Aton, Fla, USA, 1994.

[2] D. J. Montana, "The kinematics of contact and grasp," International Journal of Robotics Research, vol. 7, no. 3, pp. 1732, 1988.

[3] E. Paljug, X. Yun, and V. Kumar, "Control of rolling contacts in multi-arm manipulation," IEEE Transactions on Robotics and Automation, vol. 10, no. 4, pp. 441-452, 1994.

[4] A. B. A. Cole, J. E. Hauser, and S. S. Sastry, "Kinematics and control of multifingered hands with rolling contact," IEEE Transactions on Automatic Control, vol. 34, no. 4, pp. 398-404, 1989.

[5] S. Arimoto, M. Yoshida, M. Sekimoto, and K. Tahara, "Modeling and control of 2-D grasping of an object with arbitrary shape under rolling contact," SICE Journal of Control, Measurement, and System Integration, vol. 2, no. 6, pp. 379$386,2009$.

[6] S. Arimoto and M. Yoshida, "Modeling and control of 2D grasping under rolling contact constraints between arbitrary shapes: a Riemanniangeometry approach," Journal of Robotics, vol. 2010, Article ID 926579, 13 pages, 2010.

[7] S. Arimoto, "Dynamics of grasping a rigid object with arbitrary smooth surfaces under rolling contacts," SICE Journal of Control, Measurement, and System Integration, vol. 3, no. 3, pp. 199-205, 2010.

[8] A. Gray, E. Abbena, and S. Salamon, Modern Differential Geometry of Curves and Surfaces with Mathematics, Chapman \& Hall/CRC, Boca Aton, Fla, USA, 2006.

[9] S. Kobayashi, Differential Geometry of Curves and Surfaces, Shokabo, Tokyo, Japan, 1977, (revised in 1995 and in Japanese).

[10] M. Umehara and K. Yamada, Curves and Surfaces, Shokabo, Tokyo, Japan, 2001, (in Japanese).

[11] K. Nomizu, "Kinematics and differential geometry of submanifolds_rolling a ball with a prescribed locus of contact," Tohoku Mathematical Journal, vol. 30, pp. 623-637, 1978.

[12] M. Yoshida and S. Arimoto, "A computational model of 3D object grasping with smoothgeometry under rolling contact," in Proceedings of the 50th Annual Conference of the Society of Instrument and Control Engineers (SICE '11), pp. 919-923, 2011. 

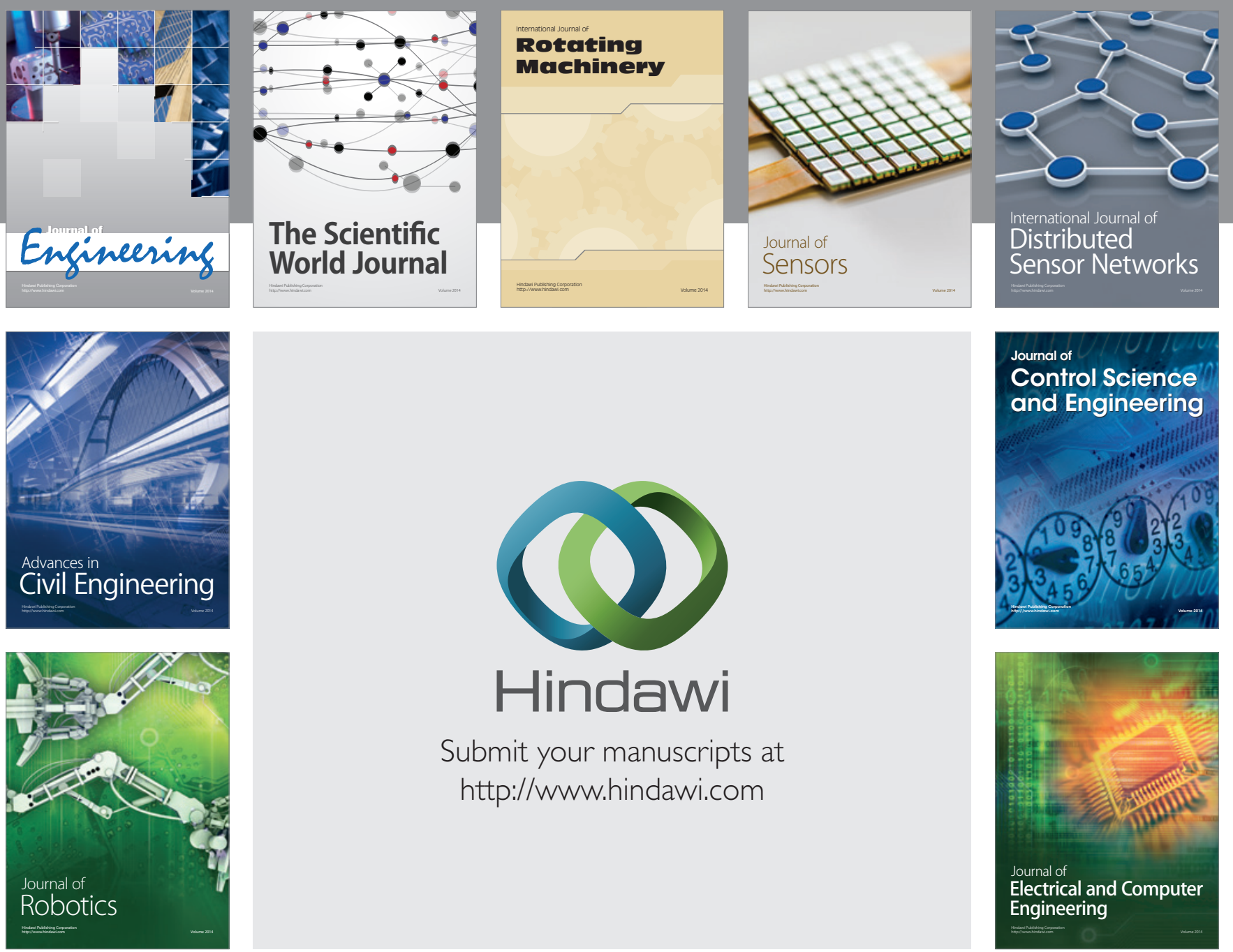

Submit your manuscripts at

http://www.hindawi.com
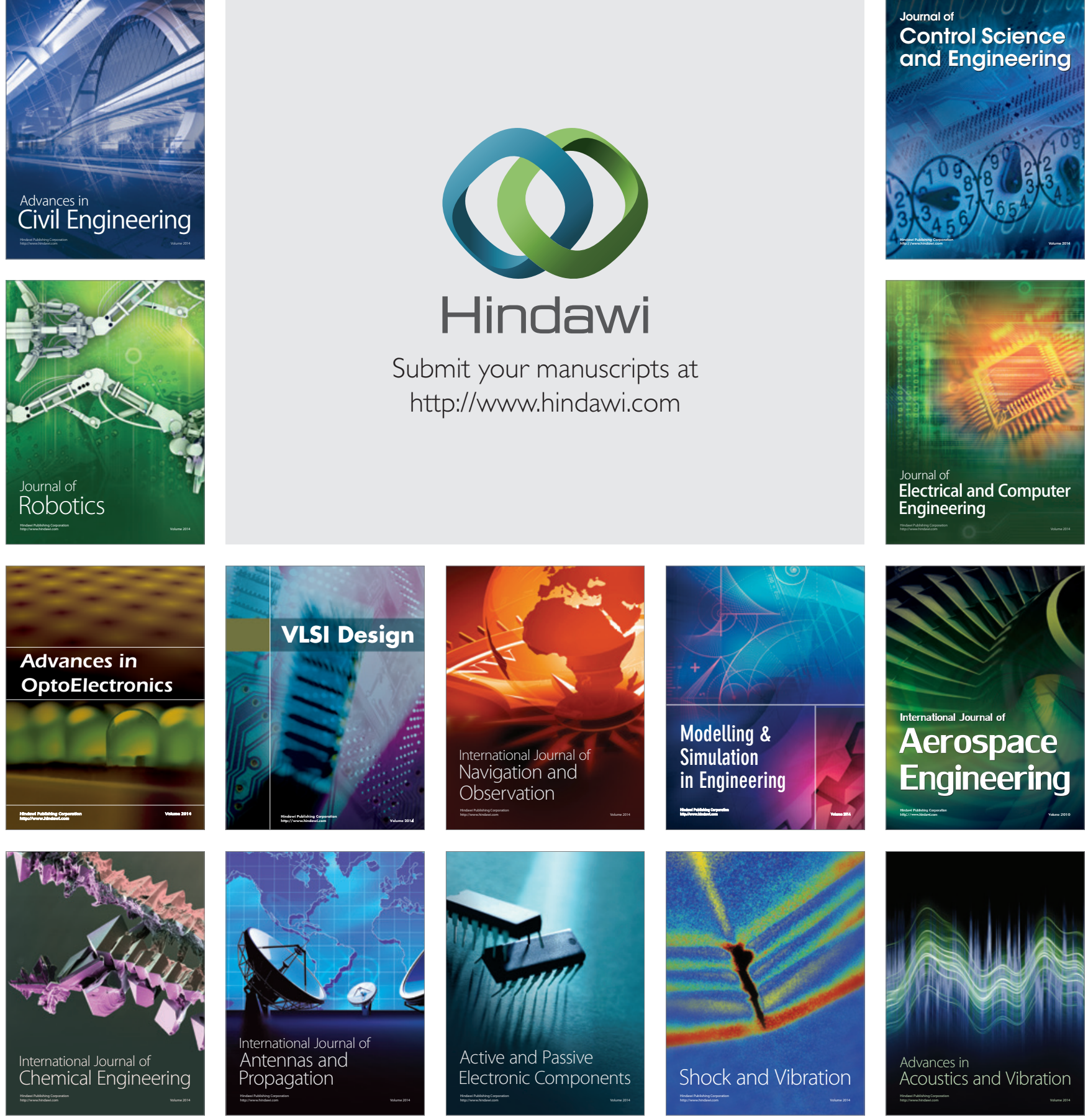